\title{
The Importance of Screening for Mild Depression in Adults with Diabetes
}

\author{
Wilhelm K $\mathrm{K}^{1,2,3}$, Reddy $\mathrm{J}^{1,3,6}$, Crawford $\mathrm{J}^{1,2}$, Robins $\mathrm{L}^{1,2,3}$, Campbell L $\mathrm{L}^{4,5}$ and Proudfoot \\ ${ }^{1}$ School of Psychiatry, Faculty of Medicine and Black Dog Institute, University of New South Wales, Randwick, NSW, Australia \\ ${ }^{2}$ St Vincent's Urban Mental Health Research Institute, St Vincent's Hospital, Sydney, NSW, Australia \\ ${ }^{3}$ Consultation Liaison Psychiatry, St Vincent's Hospital, Sydney, NSW, Australia \\ ${ }^{4}$ Diabetes Services, St Vincent's Hospital, Sydney, NSW, Australia \\ ${ }^{5}$ School of Medicine, Faculty of Medicine, University of New South Wales, Randwick, NSW, Australia \\ ${ }^{6}$ Department of Psychological and Addiction Medicine, Australian National University, Canberra ACT, Australia
}

Corresponding author: Kay Wilhelm, St Vincent's Urban Mental Health Research Institute, St Vincent's Hospital, Sydney, NSW, Australia, Tel: +61416143717; Fax: +61(02)83821402; E-mail: Kay.Wilhelm@svha.org.au

Received Date: October 12, 2016; Accepted Date: January 13, 2017; Published Date: January 18, 2017

Citation: Wilhelm K, Reddy J, Crawford J, et al. The Importance of Screening for Mild Depression in Adults with Diabetes. Transl Biomed. 2017, 8:1.

\section{Abstract}

Aim: While the impact of major depression on diabetes has gained increasing attention, the role of minor depression is less well investigated. This observational study compared three groups of adults with Type 1 (T1DM) or Type 2 (T2DM) diabetes mellitus: with me) no depressive symptoms (NODEP), ii) mild depressive symptoms (MILD) and iii) moderate-to-severe symptoms (MOD/SEVERE)-on diabetes distress, psychological distress, anxiety and somatic symptoms, quality of life and HbA1c levels.

Methods: 245 outpatients attending two hospital diabetes services ( $27 \%$ with T1DM; $73 \%$ with T2DM) completed self-report measures including patient health questionnaire (PHQ), problem areas in diabetes (PAID) scale and short form health survey (SF12). Participants were interviewed by a psychiatrist and HbA1c levels recorded.

Results: Half the sample reported depressive symptoms on the PHQ-9 (29\% in the MILD group, $21 \%$ in MOD/ SEVERE and the remaining $50 \%$ in the NODEP group). Compared to the NODEP group, MILD group participants had significantly higher levels of diabetes distress, psychological distress, anxiety and somatic symptoms and significantly lower mental-health related quality of life. Participants in the MOD/SEVERE group had significantly higher levels of diabetes distress, psychological distress, anxiety, and somatic symptoms than either of the other two groups, and significantly lower quality of life. The groups did not differ significantly on $\mathrm{HbA1c}$.

Conclusions: Mild symptoms of depression in patients with diabetes are common and are associated with higher levels of diabetes distress, psychological distress, anxiety and somatic symptoms and lower quality of life.
Addressing mild depression provides a potential avenue for preventing clinical depression in patients with diabetes.

Keywords: Diabetes; Depression; Screening; Distress; Anxiety; Subsyndromal depression

\section{Introduction}

Depression is common and burdensome in patients with diabetes. Compared to people without diabetes, depression prevalence rates are over three times higher in people with Type 1 diabetes mellitus (T1DM) and twice as high in patients with Type 2 diabetes mellitus (T2DM) [1,2]. Depression is associated with poor diabetes self-management and poorer prognosis in terms of disease severity, complications, and mortality $[3,4]$. Psychological and pharmacological interventions are effective in reducing depression in people with diabetes and lifestyle factors and some pharmacological treatments also improve glycaemic control [5]. Therefore, the importance of screening for and addressing depression in people with diabetes is recognized in the guidelines of several countries and by the International Diabetes Federation [6]. Indeed, a recent editorial stated that "clinicians must ensure that depression is recognized and treated as rigorously as the physical complications of diabetes that are generally prioritized" (p 393) [7].

Mild symptoms of depression in diabetes have been largely overlooked [8]. Mild depressive symptoms (depressive symptoms that fall short of full diagnostic criteria for major depressive disorder (MDD) or dysthymia), also known as subthreshold, subclinical, significant or subsyndromal depression, are more common in people with diabetes than MDD but less likely to be diagnosed [9]. Approximately half of those with T2DM will experience at least one episode of subthreshold depression over five years [10]. These findings, combined with evidence that subthreshold depression is a 
significant risk factor for future MDD [11], have increased attention on mild depressive symptoms in diabetes.

There is growing recognition that depression and diabetesrelated distress are overlapping but sufficiently different constructs to both be worthy of attention in view of their different properties and treatment goals $[12,13]$. The goals for 'depression', are symptom relief, suicide avoidance, relapse prevention and return to normal function, while the goals for diabetes-related distress are related to improved selfmanagement and adjustment to diabetes and $\mathrm{HbA1c}$ levels [13].

Screening for mental health issues is important but only if followed by appropriate interventions. This study aimed to contribute to the growing body of knowledge about mild symptoms of depression in people with diabetes (T1DM or T2DM) by comparing groups with three depression symptoms categories (none, mild and moderate-to-severe) on a range of variables (diabetes distress, psychological distress, anxiety and somatic symptoms, depression history, quality of life and glycosylated haemoglobin (HbA1c) levels). The aim was to screen people with established diabetes for rates of depression and consider implications for interventions in our clinical setting. This was a secondary analysis of data gathered as part of a study of relationship of gene $x$ environment interaction in a diabetes clinic [14].

It was hypothesized that (i) those with mild depressive symptoms would score higher than those without depressive symptoms on diabetes-related distress, psychological distress, anxiety, somatic symptoms and $\mathrm{HbA} 1 \mathrm{c}$ and lower on quality of life, and (ii) those with moderate-to-severe depression would score higher on these on all these measures and lower on quality of life than either of the other two groups.

\section{Method}

\section{Participants}

Participants were 245 adults with T1DM or T2DM recruited from diabetes clinics at St Vincent's Hospital and the Prince of Wales Hospital, Sydney, Australia. They were attending a clinic for people with established diabetes, often with complications. Inclusion criteria were having T1DM or T2DM, aged 18 years or over and able to read and write in English. Exclusion criteria were cognitive impairment, psychotic illness, or the occurrence of a severe life event in the past month.

\section{Measures}

The Patient health questionnaire (PHQ) is a standardized, validated 60 -item self-report diagnostic questionnaire developed as a case-finding instrument for mental disorder defined by criteria of the diagnostic and statistical manual of mental disorders-IV (DSM-IV) including major depressive disorder, general anxiety disorder, panic disorder, other anxiety disorder and bulimia and binge-eating syndromes [15]. It contains several validated, standardized measures, including:
1. The depression scale (PHQ-9): 9-item measure of depressive symptoms (PHQ-9) [16]. Categories of depression severity based on PHQ-9 scores are none-minimal (0-4), mild (5-9), moderate (10-14), moderately severe (15-19) and severe (20-27)

2. The physical symptoms (PHQ-15)-screening measure for somatization [17]

3. The generalized anxiety disorder 7-Item (GAD-7) measure of anxiety and worry [18]

The problem areas in diabetes (PAID) scale [19] is a brief and validated 20 item self-report measure of current diabetesrelated distress. Items are rated on a 0-4 Likert scale, with higher scores indicating greater distress. Internal reliability of $\alpha=0.90$ and test-retest reliability of $r=0.83$ have been reported [20].

The Kessler-10 scale (K10) [21] is a standardized and validated ten-item self-report measure of general psychological distress, depressive and anxiety symptoms during the past 4 weeks. Items rated on a 0-5 Likert scale are summed to produce a total score classifying respondents as little or no risk 0-15; medium risk 16-29 and high risk 30-50. The K10 has been shown to be sensitive in the detection of DSM-IV disorders in surveys carried out in the US and is a core measure in the annual Australian and US National Health Interview Surveys [22].

The short form health survey (SF12) [23] is a brief, validated 12 -item measure of functional health and wellbeing. A mental component score (MCS) and a physical component score (PCS) are computed, with higher scores indicating better healthrelated quality of life.

\section{Procedure}

Ethics approval was obtained from the University of New South Wales Human Research Ethics Committee (HREC Number H06/043). From July 2006 till March 2008, patients waiting to see their doctor at the Diabetes Clinic were provided information about the study by a research assistant. Those providing written consent completed a set of the study questionnaires in the waiting room. They were then interviewed in a consultation room by one of two research psychiatrists (JR or KW). This clinical interview aimed to review current and past mental health and treatment history and confirm DSM-IV axis I diagnoses and symptoms reported on the PHQ modules. HbA1c level taken on the day of consultation and duration of diabetes were obtained from clinical notes. Approximately $60 \%$ of those approached agreed to participate in the study. The main reasons for refusal were, i) "already involved with too many people in the clinic" and ii) not enough time due to work or other commitments.

A total of 274 eligible patients provided written informed consent to participate. Of these, 245 (89.4\%) completed the PHQ-9 depression measure. There were no significant differences between those with complete and missing data on the PHQ-9 in mean age or duration of diabetes illness, or proportion of males or patients with T1DM ( $p>0.05)$. The 29 
patients with incomplete data on the PHQ-9 were excluded from subsequent analyses.

\section{Data Analyses}

Data analyses were conducted using IBM SPSS Statistics- 21 . An alpha level was of 0.05 was set for all statistical tests. The sample was divided into three groups based on $\mathrm{PHQ}-9$ depression scores, using established cut-off for PHQ-9 scores: (1) 0-4 in 'No Depression' (NODEP); (2) 5-9 in 'Mild Depression' (MILD) group; 3) 10 or more in 'Moderate to Severe Depression' (MOD/SEVERE) group.

Differences between the three groups in demographic and clinical variables were determined using analyses of variance (ANOVAs) for continuous variables and chi-square goodnessof-fit analyses for categorical analyses. This was followed by a series of analyses of variance (ANOVAs), each repeated as analyses of covariance (ANCOVAs) with age as a covariate (due to significant differences between the three groups in mean age), to examine differences between the three groups in mean scores on the PAID (diabetes distress), K10 (psychological distress), GAD-7 (anxiety), PHQ-15 (somatic symptoms), SF-12 (mental and physical health related quality of life) and HbA1c. When ANOVAs indicated significant differences overall between the three groups, Bonferroni posthoc tests were conducted to determine which groups differed significantly from each other. Further chi-square goodness-offit analyses were conducted to examine differences between the three groups in the proportion of participants who had a diagnosis of major depression or any anxiety disorder, past depression, and current antidepressant use.

\section{Results}

Approximately one half of participants ( $n=123,50.2 \%)$ were in the NODEP group; 71 participants (29.0\%) were in the MILD group and 51 participants $(20.8 \%)$ were in the MOD/SEVERE group. Table 1 reports demographics and clinical variables, including $\mathrm{HbA} 1 \mathrm{c}$, for each of the three groups. There was a significant difference in mean age between groups, with the NODEP group having a significantly younger mean age $(p=0.028)$ than the MOD/SEVERE group. There were no significant differences between the three groups in rates of T1DM (26.0\% to $29.7 \%$ ), proportion of participants with at least one comorbid medical condition (high in all groups), mean duration of diabetes or mean $\mathrm{HbA1c}$ levels. However, the mean $\mathrm{HbA} 1 \mathrm{c}$ levels did increase in the expected direction across the groups (Table $\mathbf{1}$ ).

Table 1 Comparison of participants grouped by PHQ-9 depression measure scores: Demographics and health variables.

\begin{tabular}{|c|c|c|c|c|c|c|c|c|}
\hline & Group 1: No & Group 2: Mild & Group 3: Moderate/ & \multirow{3}{*}{$\begin{array}{l}F \text { or } \\
x^{2}\end{array}$} & \multirow{3}{*}{$\mathbf{p}$} & \multirow{3}{*}{$\begin{array}{l}\text { Contrast A } \\
\text { (1 vs 2) }\end{array}$} & \multirow{3}{*}{$\begin{array}{l}\text { Contrast B } \\
\text { (2 vs } 3 \text { ) }\end{array}$} & \multirow{3}{*}{$\begin{array}{l}\text { Contrast C } \\
(1 \text { vs } 3)\end{array}$} \\
\hline & $(n=123)$ & $(n=71)$ & aepression & & & & & \\
\hline & $\mathrm{M}(\mathrm{SD})$ or $\mathrm{n}(\%)$ & $\mathrm{M}(\mathrm{SD})$ or $\mathrm{n}(\%)$ & $M(S D)$ or $n(\%)$ & & & & & \\
\hline \multicolumn{9}{|l|}{ Demographics } \\
\hline Age & $59.0(13.5)$ & $57.3(13.7)$ & $52.9(13.6)$ & 3.65 & $0.028^{*}$ & NS & NS & $0.022^{*}$ \\
\hline Males & \multirow[b]{2}{*}{$67(54.5)$} & \multirow[b]{2}{*}{$34(47.9)$} & \multirow[b]{2}{*}{$33(64.7)$} & \multirow[b]{2}{*}{3.39} & \multirow[b]{2}{*}{0.183} & & & \\
\hline$(\mathrm{n}, \%)$ & & & & & & & & \\
\hline $\begin{array}{ll}\text { Married } & \text { or } \\
\text { defacto } & \\
\text { relationship } & (\mathrm{n}, \\
\%) & \end{array}$ & $61(50.4)$ & $27(39.1)$ & $25(49.0 \%)$ & 2.36 & 0.307 & & & \\
\hline $\begin{array}{l}\text { Currently } \\
\text { employed (n, \%) }\end{array}$ & $67(55.4)$ & $39(57.4)$ & $25(49.0)$ & 0.88 & 0.645 & & & \\
\hline \multicolumn{9}{|l|}{ Health variables } \\
\hline $\begin{array}{l}\text { Type } 1 \text { Diabetes } \\
(\mathrm{n}, \%)\end{array}$ & $33(29.7)$ & $19(27.9)$ & $13(26.0)$ & 0.25 & 0.885 & & & \\
\hline $\begin{array}{l}\text { Duration of } \\
\text { diabetes (years) }\end{array}$ & $13.9(11.3)$ & $12.3(11.1)$ & $15.7(13.7)$ & 0.99 & 0.374 & NS & NS & NS \\
\hline $\begin{array}{l}\mathrm{HbA} 1 \mathrm{c} \\
\mathrm{Mmol} / \mathrm{mol}(\mathrm{SD})\end{array}$ & $60(16)$ & $62(21)$ & $65(17)$ & 1.15 & 0.317 & NS & NS & NS \\
\hline (\% mean, SD) & $(7.6,1.5)$ & $(7.8,1.9)$ & $(8.1,1.6)$ & $\begin{array}{l}0.65 \\
A\end{array}$ & 0.525 & & & \\
\hline $\begin{array}{l}\text { At least one } \\
\text { other current } \\
\text { medical } \\
\text { condition }(n, \%)\end{array}$ & $94(85.5)$ & $48(80.0)$ & $32(74.4)$ & 2.68 & 0.262 & & & \\
\hline
\end{tabular}


Table 2 displays the proportion of the three groups who received a diagnosis of MDD, past depression, or any anxiety disorder, by the psychiatrist conducting a clinical interview after reviewing the PHQ self-report measures. Half those in the MOD/SEVERE group met criteria for MDD, and one participant in the MILD group; thus $10.6 \%(n=26)$ of the whole sample met criteria for MDD. Whilst there was a significant difference between the three groups in the proportion meeting DSM-IV criteria for any anxiety disorder, there were no significant differences in proportions with past depression or current antidepressant medication. (5\% of participants in MILD and MOD/SEVERE groups reported current antidepressant medication) (Table 2).

Table 2 Comparison of participants grouped by PHQ-9 depression measure scores: Psychological variables.

\begin{tabular}{|c|c|c|c|c|c|c|c|c|}
\hline & $\begin{array}{l}\text { Group 1: No } \\
\text { depression } \\
(n=123)\end{array}$ & $\begin{array}{l}\text { Group 2: Mild } \\
\text { depression } \\
(n=71)\end{array}$ & $\begin{array}{l}\text { Group 3: } \\
\text { Moderate to } \\
\text { severe } \\
\text { depression } \\
(n=51)\end{array}$ & $\mathrm{F}$ or $\mathrm{X}^{2}$ & $\mathbf{p}$ & $\begin{array}{l}\text { Contrast } A \\
\text { (1 vs 2) }\end{array}$ & $\begin{array}{l}\text { Contrast } \\
\text { (2 vs } 3 \text { ) }\end{array}$ & $\begin{array}{l}\text { Contrast } \\
\text { (1 vs } 3 \text { ) }\end{array}$ \\
\hline & $\begin{array}{l}M(S D) \text { or } n \\
(\%)\end{array}$ & $\begin{array}{l}M \text { (SD) or } \\
n(\%)\end{array}$ & $\begin{array}{l}M(S D) \text { or } n \\
(\%)\end{array}$ & & & & & \\
\hline PAID & $16.2(13.8)$ & $29.3(20.4)$ & $45.3(29.1)$ & 29.79 & $<0.0001^{* * *}$ & $0.001^{* *}$ & $0.001^{* *}$ & $<0.0001^{* * *}$ \\
\hline (Diabetes distress) & & & & $27.62 \mathrm{~A}$ & $<0.0001^{* * *}$ & & & \\
\hline $\begin{array}{l}\text { PHQ-9 } \\
\text { (Depression) }\end{array}$ & $1.7(1.4)$ & $6.5(1.3)$ & $14.9(4.2)$ & $\begin{array}{l}608.18 \\
580.51 \mathrm{~A}\end{array}$ & $\begin{array}{l}<0.0001^{* * *} \\
<0.0001^{* * *}\end{array}$ & $<0.0001^{* * *}$ & $<0.0001^{* * *}$ & $<0.0001^{\star * *}$ \\
\hline GAD-7 (Anxiety) & $3.7(2.1)$ & $5.7(2.5)$ & $9.3(3.0)$ & $\begin{array}{l}52.83 \\
50.47 \mathrm{~A}\end{array}$ & $<0.0001^{* * *}$ & $<0.0001^{* * *}$ & $<0.0001^{* * *}$ & $<0.0001^{* * *}$ \\
\hline $\begin{array}{l}\text { PHQ-15 (Somatic } \\
\text { symptoms) }\end{array}$ & $3.1(2.4)$ & $5.8(3.3)$ & $9.0(3.8)$ & $\begin{array}{l}57.12 \\
58.43 \mathrm{~A}\end{array}$ & $\begin{array}{l}<0.0001^{* * *} \\
<0.0001^{* * *}\end{array}$ & $<0.0001^{* * *}$ & $<0.0001^{* * *}$ & $<0.0001^{* * *}$ \\
\hline $\begin{array}{l}\text { K10 } \\
\text { (Psychological } \\
\text { distress) }\end{array}$ & $12.7(3.1)$ & $17.7(4.7)$ & $28.8(8.1)$ & $\begin{array}{l}140.73 \\
134.91 \mathrm{~A}\end{array}$ & $\begin{array}{l}<0.0001^{* * *} \\
<0.0001^{* * *}\end{array}$ & $<0.0001^{* * *}$ & $<0.0001^{* * *}$ & $<0.0001^{* * *}$ \\
\hline $\begin{array}{l}\text { SF-12 mental } \\
\text { QoL\# }\end{array}$ & $53.8(8.3)$ & $47.1(8.4)$ & $36.6(11.0)$ & $\begin{array}{l}61.8 \\
58.28 \mathrm{~A}\end{array}$ & $\begin{array}{l}<0.0001^{* * *} \\
<0.0001^{* * *}\end{array}$ & $<0.0001^{* * *}$ & $<0.0001^{* * *}$ & $<0.0001^{\star * *}$ \\
\hline $\begin{array}{l}\text { SF-12 physical } \\
\text { QoL\# }\end{array}$ & $44.7(10.3)$ & $40.9(11.6)$ & $39.6(10.6)$ & $\begin{array}{l}4.92 \\
8.74 \mathrm{~A}\end{array}$ & $\begin{array}{l}0.008^{* *} \\
<0.000^{* \star *}\end{array}$ & Trend 0.064 & NS & $0.019^{*}$ \\
\hline $\begin{array}{l}\text { Major depression } \\
\text { (clinician } \\
\text { diagnosed) }\end{array}$ & $0(0 \%)$ & $1(1.4 \%)$ & $25(49.0 \%)$ & 100.25 & $<0.0001^{* * *}$ & & & \\
\hline $\begin{array}{l}\text { Any anxiety } \\
\text { disorder }(\mathrm{PHQ})\end{array}$ & $2(1.6 \%)$ & $7(9.9 \%)$ & $14(28.0 \%)$ & 46.22 & $<0.0001^{* * *}$ & & & \\
\hline $\begin{array}{l}\text { Past depression } \\
(\mathrm{n}, \%)\end{array}$ & $29(26.9 \%)$ & $16(26.7 \%)$ & $16(37.2 \%)$ & 1.94 & NS & & & \\
\hline $\begin{array}{l}\text { Current } \\
\text { antidepressant } \\
\text { medication }(n, \%)\end{array}$ & $8(7.3 \%)$ & $3(5.1 \%)$ & $2(4.9 \%)$ & 0.41 & NS & & & \\
\hline
\end{tabular}

Table 2 reports results of a series of ANOVAs, each repeated as an ANCOVA with age as a covariate, examining differences between the three groups in means of psychological selfreport measures. There were significant differences between the groups in diabetes distress (PAID), psychological distress (K10), anxiety (GAD-7) and somatic symptoms (PHQ-15), with each measure showing a similar three-tier effect in severity from the MOD/SEVERE group to the NODEP group (Figure 1). 


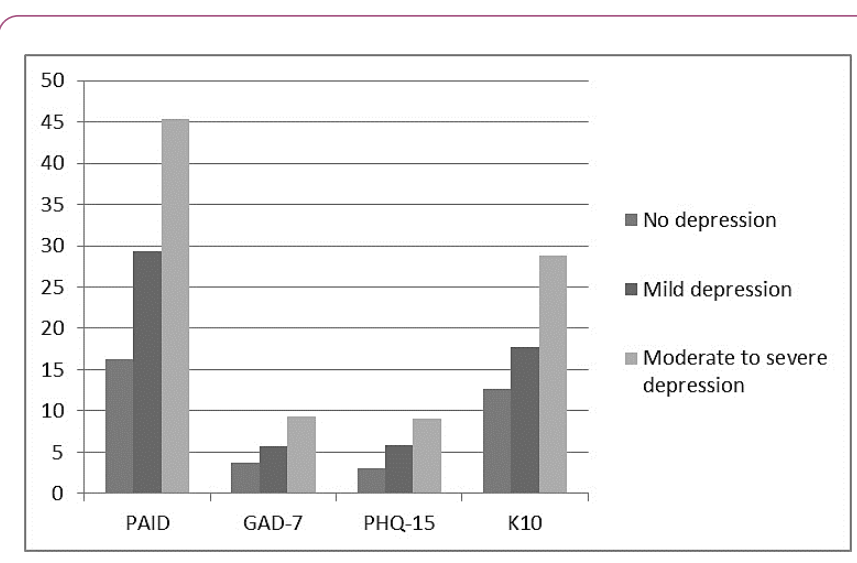

Figure 1 Means of the PAID (diabetes distress) GAD-7 (anxiety), PHQ-15 (somatic symptoms) and K10 (psychological distress) in patients grouped by $\mathrm{PHQ}-9$ depression scores.

Post-hoc Bonferroni contrasts (Table 1) found significant differences between the groups on all psychological measures $(p<0.01$ to $p<001)$. That is, the MILD group had higher means than the NODEP group in diabetes distress (PAID), psychological distress (K10), anxiety (GAD-7) and somatic symptoms (PHQ-15), and the MOD/SEVERE group had higher means than the MILD group on each of these measures.

Table 2 also presents results of ANOVAs, again repeated as ANCOVAs with age as a covariate, examining differences between the three groups in health-related quality of life (HRQoL), assessed by the SF-12 Mental Component Score (MCS-12: mental HRQoL) and Physical Component Score (PCS-12: physical HRQoL). There were significant differences between the groups in the means of both the MCS-12 and PCS-12. Bonferroni post-hoc contrasts revealed that the MILD group had a significantly lower MCS-12 (indicating lower mental HRQoL) than the NODEP group $(p<0.001)$ and the MOD/SEVERE group had significantly lower MCS-12 than the MILD group $(p<0.001)$. For PCS-12, the MOD/SEVERE group had significantly lower PCS-12 than the NODEP group $(p<0.05)$ and there was a non-significant trend $(p=0.064)$ for the MILD group to have a lower PCS-12 than the NODEP group.

\section{Discussion}

As hypothesized, there was a consistent three-tiered pattern of symptom severity across the three groups (none, mild and moderate/severe depression) for all psychological self-report measures used (i.e. higher levels of diabetes distress, psychological distress, anxiety and somatic symptoms and significantly lower mental health-related quality of life), all differences between groups being statistically significant.

Whilst there were no significant differences in $\mathrm{HbA1c}$ levels, there was a trend for the mean $\mathrm{HbA} 1 \mathrm{c}$ levels to rise across the three groups. This is consistent with previous reports of more robust findings of the relationship between $\mathrm{HbA} 1 \mathrm{c}$ levels and diabetes distress than depression [12,24,25]. This may be because the association between depression and glycaemic control is mediated by several pathways [21] and/or the impact of depression on glycaemic control can vary with other concurrent stressors and needs [20].

The prevalence rates of depressive symptoms found in this study $(29 \%$ with mild symptoms and $21 \%$ with moderate-tosevere symptoms, including $11 \%$ who met DSM-IV criteria for MDD) are consistent with previous findings that mild depressive symptoms are more common than MDD in people with diabetes [9]. They are also consistent with a large Australian survey of people with T1DM or T2DM, where only $13 \%$ reported that had received a diagnosis of depression [26] despite $22 \%$ to $35 \%$ of respondents reporting clinically significant depressive symptoms. Interestingly, we found no differences between the three groups in the proportion with past depression (reported by a quarter to a third in each group). Only $5 \%$ of patients with either mild or moderate/ severe symptoms of depression reported currently taking antidepressant medication. Whilst it is not known how many participants were currently receiving psychological treatment, this suggests undertreatment of depression in the study sample.

The high prevalence of mild depressive symptoms amongst patients with diabetes is important because of the demonstrated association with lower quality of life and higher levels of diabetes distress, psychological distress, anxiety, and somatic symptoms. Further, subthreshold depression has previously been found to be associated with difficulties in selfmanaging diabetes [27] and to pose a risk for future MDD [11].

We have demonstrated that it is possible to screen for depression across the severity spectrum in a busy diabetes service. We consider that screening for distress and depression in diabetes is feasible and our research is consistent with previous recommendations to screen for both depression (using the PHQ-9) and diabetes distress (using the PAID or Diabetes Distress Scale) [26].

We note a recent call for a single, continuous construct of 'emotional distress' to be used to assess and manage distress in patients with diabetes and reduce any confusion between diabetes distress, depression and distress from life stressors [28]. Whilst such an approach is conceptually concise and has several advantages outlined by the authors, we have listed below several forms that mild depression could take, each with different management implications. We would therefore favour screening for both depression and diabetes distress in patients with diabetes.

We agree that screening for emotional distress in and of itself is not sufficient to improve outcomes for diabetes patients [12,29], and is only helpful if followed up by appropriate interventions. This should include early intervention to treat mild depression as a means of preventing future episodes of depression and improving quality of life and self-management of diabetes. We speculate that mild depression could represent a number of conditions including evolving or partially treated major depression, chronic unhappiness and demoralization, comorbid anxiety disorders, eating disorders, personality disorders, substance use or pain. 
All these are going to impact on diabetes control so that mild depression is both important in its own right but also a marker of a need for further diagnostic clarification.

Given the large numbers affected, time and costeffectiveness are key issues. Screening processes involving mailed letters, research assistants and interviews with psychologists may not be cost-effective [30]. As diabetes distress is significantly correlated with impaired glycaemic control, depression severity and psychological distress, and is highly prevalent in people with diabetes with a history of depression [31], it is recommended that treatments for depression in people with diabetes also address diabetesrelated distress [32]. Thus, future research could examine costeffective methods of both mental health screening and easily disseminated tailored self-help interventions for mild depression in diabetes and/or diabetes distress that investigate the impact on both diabetes self-management (including impact on $\mathrm{HbA} 1 \mathrm{c}$ ) and on current mood state and future depressive episodes.

There is growing interest in face-to-face and online interventions in this area. Some recent large trials have demonstrated that even low-intensity interventions can be effective at reducing both mild depressive symptoms and diabetes distress. A study [32] examining three interventions (psychoeducation, physical exercise and 'enhanced treatment as usual') for adults with T2DM and subsyndromal depression found all three were equally effective in improving depressive symptoms, diabetes distress, self-management of diabetes and quality of life, with improvements sustained at one year follow-up. Another study for adults with T2DM and diabetes distress [33] found three different interventions (all Internetbased with telephone contact) all significantly improved diabetes distress, emotional symptoms and self-management. Diabetes-specific cognitive behavioral group therapy for people with diabetes and subclinical depression [34] and webbased guided CBT for people with T1DM or T2DM and mild to moderate depression [35] have also reported significant reductions in both depression and diabetes distress. Further trials utilizing Internet and mobile technologies for the mental health of people with diabetes are currently being conducted [36], including our own study using a local iCBT study for depression in people with diabetes [37]. A recent report of an online diabetes self-management program, of the sort which could compliment depression resources reported 'small but significant' benefits as a stand-alone program [38] but points to the need for a mixture of face-to-face and online resources targeted to individual needs.

It has been suggested that diabetes patients with mild symptoms of depression or diabetes distress can be highly responsive to clinical staff attention, care and support [32,33] and even minimal, low-cost, web-based interventions can improve depressive symptoms, diabetes distress and selfmanagement in this population [33]. While it is unlikely that any single approach for people with mild depression in diabetes will be universally useful, we submit that identifying these people as an 'at risk' group requiring further assessment and appropriate interventions is as important as identifying and managing those 'at risk' of physical complications of diabetes [7].

A recent review of treatment and health-care delivery for depression and diabetes [13] suggested a stepped approach, with step 1 being for mild depression (or subthreshold depression causing impairment). They suggest that after suicide and acute distress have been excluded, this approach is best carried out with a flexible patient-centered approach which can involve face-to-face and internet-based elements, but is likely to contain both approaches for most people. This is all consistent with the studies noted above.

In term of limitations of this study, the sample of outpatients from a specialist hospital diabetes service had high levels of medical comorbidity and may not be representative of patients with diabetes in primary care [39]. However, the issues raised by this study still have broad relevance in pursuing an integrated care approach. Further limitations include failure to collect details of non-participants in a busy outpatient setting and lack of assessment of diabetes self-care and psychological treatments received.

\section{Conclusions}

Mild symptoms of depression were reported by $29 \%$ of the sample of patients with T1DM or T2DM and associated with significantly higher levels of diabetes distress, psychological distress, anxiety and somatic symptoms and significantly lower mental-health related quality of life, relative to those without symptoms of depression. Screening for mild depressive symptoms in diabetes can create the opportunity to provide such patients with low-cost early interventions to improve quality of life and prevent future MDD. Future studies could examine the utility and cost-effectiveness of such an approach, and with longer term follow-up could evaluate the impact of such interventions in preventing later MDD and improving diabetes-related behavior, and eventually glycaemic control.

\section{Acknowledgements}

Adam Finch, Elaine Royal, Jennifer Nicholas, Lucinda Wedgwood, Tara Showy in, Jennifer Siegel, for data collection and patient telephone support and follow-up. We acknowledge support from NHMRC Grants 222708 and 230802 and an Infrastructure Grant from Centre for Mental Health, NSW Ministry of Health.

Funding: This study was supported by Australian Government National Health and Medical Research Council (NHMRC) Grants 222708, 230802, 157209 and by an Infrastructure Grant from the Centre for Mental Health, NSW Department of Health.

\section{References}

1. Anderson RJ, Freedland K, Clouse RE, Lustman PJ (2001) The prevalence of comorbid depression in adults with diabetes. Diabetes Care 24: 1069-1078. 
2. Roy T, Lloyd C (2012) Epidemiology of depression and diabetes: A systematic review. J Affec Disord 142: S8-S21.

3. Egede LE, Niertert PJ, Zheng D (2005) Depression and all-cause and coronary heart disease mortality among adults with and without diabetes. Diabetes Care 28: 1339-1345.

4. Gonzalez JS, Safren A, Delhanty LM, Cagliero E, Wexler DJ, et al. (2008) Symptoms of depression prospectively predict poorer self-care in patients with Type 2 diabetes. Diabetic Medicine 25: 1102-1107.

5. Baumeister H, Hutter N, Benger J (2014) Psychological and pharmacological interventions for depression in patients with diabetes mellitus: an abridged Cochrane review. Diabetic Med 31: 773-786.

6. International Diabetes Federation (2012) Clinical Guidelines Taskforce. Global Guideline for Type 2 Diabetes. IDF Communications: Brussels.

7. The Lancet Diabetes Endocrinology (2015) Poor mental health in diabetes: Still a neglected comorbidity. Lancet Diabetes Endocrinol 3: 393.

8. Pibernik-Okanovic $\mathrm{M}$, Ajdukovik D, Loverncic MV, Hermanns $\mathrm{N}$ (2011) Does treatment of subsyndromal depression improve depression and diabetes related outcomes: protocol of a randomised controlled comparison of psycho-education, physicl exercise and treatment as usual. Trials 12: 17.

9. Li C, Ford E, Zhao G, Ahluwalia IB, Pearson WS, et al. (2009) Prevalence and correlates of undiagnosed depression among U.S. adults with diabetes: The Behavioral Risk Factor Surveilaance System, 2006. Diabetes Res Clin Pract 83: 268-279.

10. Schmitz N, Gariepy G, Smith KJ, Clyde M, Malla A, et al. (2014) Recurrent subthreshold depression in Type 2 diabetes: An important risk factor for poor outcomes. Diabetes Care 37 970-978.

11. Cuijpers P, Smit F (2004) Subthreshold depression as a risk indictor for major depressive disorder: A systematic review of prospective studies. Acta Scandinavica Psychiatrica 109: 325-31.

12. Snoek FJ, Bremmer MA, Hermanns N (2015) Contructs of depression and distress in diabetes: time for an appraisal. Lancet Diabetes Endocrinol 3: 450-460.

13. Petrak F, Baumeister H, Skinner TC, Brown A, Holt RIG (2015) Depression and diabetes: treatment and health-care delivery. Lancet Diabetes Endocrinol 3: 472-485.

14. Wilhelm K, Gillis I, Reddy J, Mitchell PB, Campbell L, et al. (2012) Association between serotonin transporter promoter polymorphisms and psychological distress in a diabetic population. Psychiatry Res 200: 343-8.

15. Dies-Quevedo C, Rangil T, Sanchez-Planell L, Kroenke K, Spitzer R (2001) Validation and utility of the Patient Health Questionnaire in diagnosing mental disorders in 1003 general hospital Spanish inpatients. Psychosom Med 63: 679-686.

16. Kroenke K, Spitzer R, Williams J (2001) The PHQ-9: Validity of a brief depression severity measure. J Gen Inter Med 16: 606-613.

17. Kroenke K, Spitzer R, Williams J (2002) The PHQ-15: Validity of a new measure for evaluating severity of somatic symptoms. Psychosom Med 64: 258-266.

18. Spitzer RL, Kroenke K, Williams JBW, Löwe B (2006) A brief measure for assessing generalized anxiety disorder: The GAD-7. Arch Intern Med 166: 1092-1097.
19. Polonsky WH, Anderson BJ, Loher PA, Welch G, Jacobson AM, et al. (1995) Assessment of diabetes-related distress. Diabetes Care 18: 754-760.

20. Welch G, Alan MJ, William HP (1997) The problem areas in diabetes scale: An evaluation of its clinical utility. Diabetes Care 20: 760.

21. Kessler R, Andrews G, Colpe L, Hiripi E, Mroczek DK, et al. (2002) Short screening scales to monitor population prevalences and trends in non-specific psychological distress Psychol Med 32: 959-976.

22. Furukawa $T$, Kessler $R$, Slade $T$, Andrews $G$ (2003) The performance of the $\mathrm{K} 6$ and $\mathrm{K} 10$ screening scales for psychological distress in the Australian National Survey of Mental Health and Well-Being. Psychol Med 33: 357-362.

23. Ware JE Jr, Kosinski M, Keller SD (1996) A 12-item short-form health survey: Construction of scales and preliminary tests of reliability and validity. Med Care 34: 220-233.

24. Bot M, Pouwer F, de Jonge P, Tack CJ, Geelhoed-Duijvestijn PH, et al. (2013) Differential associations between depressive symptoms and glycaemic control in outpatients with diabetes. Diabetic Med 30: e115-e122.

25. Fisher L, Mullan JT, Arean P, Glasgow RE, Hessler D, et al. (2010) Diabetes distress but not clinical depression or depressive symptoms is associated with glycaemic control in both crosssectional and longitudinal analyses. Diabetes Care 33: 1935-5548.

26. Speight J, Browne J, Holmes-Truscott E, Hendrieckx C, Halliday J, et al. (2011) Diabetes MILES Australia 2011 Report. Canberra.

27. Gonzalez JS, Delahanty LM, Safren SA, Meigs JB, Grant RW (2008) Differentiating symptoms of depression from diabetesspecific distress: relationships with self-care in diabetes. Diabetologica 51: 1822-1825.

28. Fisher L, Gonzalez JS, Polonsky WH (2014) The confusing tale of depression and distress in patients with diabetes: A call for greater clarity and precision. Diabetic Med 31: 764-772.

29. Pouwer $F$ (2009) Should we screen for emotional distress in type 2 diabetes mellitus? Nat Rev Endocrinol 5: 665-671.

30. Fleer J, Tovcote KA, Keers JC, Links TP, Sanderman R, et al. (2013) Screening for depression and diabetes-related distress in a diabetes outpatient clinic. Diabetic Med 30: 88-94.

31. Reddy J, Wilhelm K, Campbell L (2013) Putting PAID to diabetesrelated distress: The potential utility of the problem areas in diabetes (PAID) scale in patients with diabetes. Psychosomatics 54: 44-51.

32. Pibernik-Okanovic $M$, Hermanns $N$, Ajdukovic $D$, Kos J, Prašek $M$, et al. (2015) Does treatment of subsyndromal depression improve depression-related and diabetes-related outcomes? A randomized controlled comparison of psychoeducation, physical exercise and enhanced treatment as usual. Trials 16: 305.

33. Fisher L, Hessler D, Glasgow RE, Arean PA, Masharani U, et al. (2013) Redeem: A pragmatic trial to reduce diabetes distress. Diabetes Care 36: 2551-2558.

34. Hermanns N, Schmitt A, Gahr A, Herder C, Nowotny B, et al. (2015) The effect of a diabetes-specific cognitive behavioural treatment program (DIAMOS) for patients with diabetes and subclinical depression: Results of a randomized controlled trial. Diabetes Care 38: 551-560. 
35. van Bastelaar KM, Pouwer F, Cuijpers P, Riper H, Snoek FJ (2011) Web-based depression treatment for type 1 and type 2 diabetic patients: A randomized, controlled trial. Diabetes Care 34: 320-325.

36. Clarke J, Vatiliotis V, Verge CF, Holmes-Walker J, Campbell LV, et al. (2015) A mobile phone and web-based intervention for improving mental well-being in young people with type 1 diabetes: Design of a randomized controlled trial. JMIR Res Protocol 4: e50.

37. Robins L, Newby J, Wilhelm K, Smith J, Fletcher T, et al. (2015) Internet-delivered cognitive behavior therapy for depression comorbid with diabetes: Study protocol for a randomized controlled trial. BMJ Open Diabetes Res Care 3: e000144.

38. Lorig K, Ritter PL, Turner RM, English K, Laurent DD, et al. (2016) A diabetes self-management program: 12-month outcome sustainability from a nonreinforced pragmatic trial. J Med Internet Res 18: e322.

39. Stoop CH, Nefs G, Pop VJ, Wijnands-van Gent CJ, Tack CJ, et al. (2014) Diabetes-specific emotional distress in people with Type 2 diabetes: A comparison between primary and secondary care. Diabetic Med 31: 1252-1259. 